Slavica

bruxellensia

\section{Slavica bruxellensia}

Revue polyphonique de littérature, culture et histoire

slaves

\section{$4 \mid 2009$}

Espaces slaves / espaces belges

\title{
La réception de Maeterlinck dans la Jeune Pologne
}

Voix de trois lecteurs.

\section{Dariusz Dziurzyński}

Traducteur : Alexandra Dufour

\section{OpenEdition \\ Journals}

Édition électronique

URL : http://journals.openedition.org/slavica/264

DOI : $10.4000 /$ slavica.264

ISSN : 2034-6395

Éditeur

Université libre de Bruxelles - ULB

Édition imprimée

Pagination : 7-21

ISSN : 2031-7654

\section{Référence électronique}

Dariusz Dziurzyński, «La réception de Maeterlinck dans la Jeune Pologne », Slavica bruxellensia [En ligne], 4 | 2009, mis en ligne le 15 octobre 2009, consulté le 01 mai 2019. URL : http:// journals.openedition.org/slavica/264; DOI : 10.4000/slavica.264

Ce document a été généré automatiquement le 1 mai 2019.

\section{(c) (i) $\odot$}

Les contenus de Slavica bruxellensia sont mis à disposition selon les termes de la Licence Creative Commons Attribution - Pas d'Utilisation Commerciale - Pas de Modification 3.0 France. 


\section{La réception de Maeterlinck dans la Jeune Pologne}

Voix de trois lecteurs.

Dariusz Dziurzyński

Traduction : Alexandra Dufour

\section{NOTE DE L'ÉDITEUR}

La langue originelle de cet article est le polonais

1 Les études littéraires polonaises se sont relativement peu intéressées à l'œuvre de Maurice Maeterlinck, mais elles ont toutefois produit des analyses brillantes et très érudites. Maciej Żurowski a abordé le problème de la réception de la poésie de jeunesse de l'auteur belge au tournant des $\mathrm{XIX}^{\mathrm{e}}$ et $\mathrm{XX}^{\mathrm{e}}$ siècles en Pologne ${ }^{1}$. Maria Stykowa s'est penchée quant à elle sur la réception théâtrale de l'œuvre de celui-ci sur les scènes de la Jeune Pologne ${ }^{2}$. Ses recherches se basent et sont partiellement inspirées des constatations d'Irena Sławińska. Cette chercheuse fut la première à démontrer l'influence du dramaturge sur la conscience et la pratique théâtrale de Stanisław Wyspiański et de Stanisław Przybyszewski³.

2 Les chercheurs polonais ont toutefois brillé par leur présence sur la scène internationale. Aniela Słomkowska a analysé les rôles du paysage dans le drame maeterlinckien ${ }^{4}$ et Włodzimierz Szturc a publié à Gand un livre sur la dialectique des catégories de l'intériorité et de l'extériorité dans la création littéraire et dans les réflexions philosophiques du célèbre auteur ${ }^{5}$. Le périodique belge « ANNALES DE LA FONDATION MAURICE MAETERLINCK ", fondé en 1955 pour étudier la résonance mondiale de la création de l'auteur des Serres chaudes, montre très bien l'apport des chercheurs polonais à la reconnaissance européenne de la réception de son œuvre. Y figurent quatre articles, écrits par des spécialistes polonais de la littérature, consacrés soit à la mise en perspective de la réception de l'œuvre de Maeterlinck, soit à des tentatives de relecture de certaines questions essentielles ${ }^{6}$. 
3 La création du symboliste belge a déjà émerveillé, avec une même intensité, plusieurs générations de chercheurs polonais tout comme l'œuvre d'Émile Zola, son prédécesseur, l'autre "maréchal des lettres européennes » ${ }^{*_{7}} \mathrm{du} \mathrm{XIX}^{\mathrm{e}}$ siècle.

4 Le thème de la réception de l'œuvre de Maeterlinck dans la Jeune Pologne est un sujet très complexe. Les difficultés sont, dans un certain sens, occasionnées par Maeterlinck lui-même. La création de celui-ci s'inscrit en effet dans quatre genres littéraires différents (la nouvelle, la poésie, le théatre et l'essai). De plus, les multiples facettes de son talent offrent aux lecteurs étrangers un choix très varié. Les études actuelles sur la réception de l'œuvre de Maeterlinck en Pologne se situent principalement sur deux plans : la réception de sa poésie et celle de ses pièces de théâtre (et de leurs réalisations scéniques). La nouveauté dans le domaine de la réception littéraire devrait donc, dans ce cas, reposer sur l'appropriation, par la critique nationale et le grand public polonais, des nouvelles de l'auteur belge, mais surtout de ses essais.

Il ne faut cependant pas oublier que la création de l'auteur des Aveugles a attiré l'attention de peintres et de graphistes ainsi que celle de compositeurs. À son époque, Maeterlinck était donc une nouvelle source d'inspiration pour les illustrateurs de livres $^{8}$ ou les peintres symbolistes tels que Fernand Khnopff et Carlos Schwab9. On le sait, il a aussi été celui qui a inspiré la réforme du drame musical et de l'opéra (il suffit de citer l'exemple de Pelléas et Mélisande de Claude Debussy).

6 Le phénomène " Maeterlinck » dépasse donc résolument le domaine littéraire et pousse à entreprendre des recherches interdisciplinaires, ce qui confirme, d'une manière presque romantico-symboliste, l'idéal de l'unité des arts. L'appropriation par la littérature de l'écriture de Maeterlinck peut déjà être un objet de réception en soi. Toutefois, derrière l'analyse de la résonance générale de son œuvre, dans un esprit de réception comparatiste, elle révèle de manière décisive l'unité d'un «moment historique » dans lequel deux facteurs, la littérature et l'art, constituèrent une entité complexe. Parmi les premières études interdisciplinaires sur les liens de l'œuvre de Maeterlinck avec la musique, nous trouvons les conclusions de Stykowa sur l'accompagnement musical des mises en scènes polonaises de Pelléas et Mélisande et de L'oiseau bleu ${ }^{10}$. Les premières réflexions synthétiques sur la peinture sont dues à la plume de Wiesław Juszczak qui a découvert l'influence du célèbre dramaturge sur l'œuvre, entre autres, d'Edward Okun, Witold Wojtkiewicz et Olga Boznańska ${ }^{11}$. Nous ignorons toujours cependant si derrière l'accueil réservé à Maeterlinck-écrivain par la critique polonaise ne se cachait pas la réception du symbolisme idéaliste des peintres belges et français, qui, comme le démontrent les recherches de Jean-David Jumeau-Lafond, considéraient l'auteur de La Princesse Maleine comme l'un des écrivains qui a le plus touché leur imagination ${ }^{12}$. Dans cette hypothèse, il serait alors possible de lier des phénomènes en apparence très distants en une entité interdisciplinaire : d'une part, la popularité scénique et la traduction des œuvres de Maeterlinck et, d'autre part, les expositions du Salon Krywult ou de « CHIMERA ». Comme le montre Grzegorz BĄbiak ${ }^{13}$, ces expositions ont introduit les œuvres des symbolistes étrangers dans la vie culturelle nationale. La question de la fidélité des illustrations dans les périodiques littéraires et artistiques phares de la Jeune Pologne pourraient également bénéficier ici d'une explication génétique ${ }^{14}$.

7 Dans cette de recherche sur la réception, c'est Wyspiański qui a fourni les éléments les plus notoires. C'est lui qui a créé, comme l'a rappelé Przybyszewski bien des années après, les dessins floristiques en marge de l'édition parisienne des Serres chaudes et qui a proposé d'en publier la version polonaise dans sa propre conception graphique ${ }^{15}$. Il n'est jamais 
parvenu à réaliser ce dessein mais l'idée même suggère une perspective qui permet d'inclure Wyspiański parmi les meilleurs illustrateurs étrangers des livres poétiques de Maeterlinck au nombre desquels il faut compter Charles Doudelet, à qui l'on doit une pleine page d'illustrations pour Quinze Chansons, et Georges Minne, qui esquissa un en-tête pour le premier tome de 1889.

Il existe un autre problème encore concernant la réception à l'étranger de l'œuvre de Maeterlinck. Il implique non pas la polymorphie du talent de l'écrivain ni la résonance interdisciplinaire de ses œuvres mais, cette fois, la nature même du phénomène cognitif qu'est la réception dans les analyses comparatistes.

Comme l'a montré Hans Robert Jauss, la question de la réception internationale de la création littéraire constitue une partie conséquente du processus de communication littéraire ${ }^{16}$. Il instaure, entre la production littéraire et sa réception, un dialogue social et intersubjectif dans lequel ces deux facteurs jouent un rôle équivalent. La réception, vue sous cet angle, n'est pas simplement une assimilation passive d'un phénomène culturel étranger, mais une réponse sélective et active à ce qui est extérieur. Le récepteur littéraire est défini à travers une réalité temporelle et environnementale concrète. Sa conscience n'a une signification effective qu'en tant que maillon dans la chaîne des concrétisations historiques d'un phénomène donné. À la lumière des écrits de Jauss, la question de la réception de l'œuvre de Maeterlinck dans la Jeune Pologne doit donc apparaitre comme une concrétisation unique, ponctuelle, de la problématique que constitue la destinée internationale de la création de Maeterlinck. Cette concrétisation se soumet en outre à une prédétermination extérieure.

10 C'est pourquoi, pour les développements ultérieurs, il sera important d'analyser non seulement la façon dont les œuvres de Maeterlinck ont été mises à la disposition du lecteur et du spectateur polonais, mais aussi le moyen par lequel l'évolution littéraire de la Jeune Pologne, pourtant régie par ses propres règles, a influencé la perception de ses œuvres. En bref, ce n'est pas seulement l'œuvre qui est essentielle ici, mais également l'impact des circonstances locales sur elle. Jusqu'à un certain point, Maeterlinck a façonné une nouvelle ère dans la littérature polonaise. Cependant, à partir d'un certain moment, cette nouvelle époque déjà solide et formée, influença de façon décisive le Maeterlinck polonisé et profila son image.

11 Il est possible d'illustrer cette dialectique de l'œuvre et de l'époque par un grand nombre de faits en inscrivant la réception de l'œuvre de Maeterlinck dans le schéma du développement littéraire de la Jeune Pologne. Dès le premier coup d'œil, une certaine régularité apparait: la présence de l'écrivain belge correspond à une période de changements dans l'évolution de l'époque et influe aussi bien sur la pratique créatrice habituelle que sur l'élaboration de la conscience littéraire et, plus généralement, philosophique. D'un point de vue général, nous pouvons distinguer trois moments principaux dans la réception de Maeterlinck dans la Jeune Pologne. Chacun de ces moments correspond à une phase : le début, la maturité et la période de réforme interne de la formation de la Jeune Pologne. Par conséquent, nous pouvons parler d'un Maeterlinck des temps de "la révolte moderniste » (début des années 1890), de celui de la période culminante (fin des années 1890) et enfin d'un Maeterlinck de la crise esthétique et idéologique, annonciatrice de la campagne de liquidation de la Jeune Pologne (moitié de la première décennie $\mathrm{du} \mathrm{XX}^{\mathrm{e}}$ siècle). Il se fait qu'au tournant du siècle, chacun des maillons de la chaîne de la réception est associé au nom d'un autre coryphée de la littérature polonaise: dans le premier des cas, il s'agit de Zenon Przesmycki, dans le 
deuxième cas, de Przybyszewski, et de Stanisław Brzozowski dans le troisième cas. Parmi le groupe polonais de lecteurs de Maeterlinck, ce sont ces personnages qui sont les plus dignes d'être présentés à un interlocuteur étranger.

Ajoutons encore que les trois aspects les plus importants de la réception littéraire (la traduction, la critique et le théâtre) seront analysés conjointement dans cette esquisse.

\section{La voix de Zenom Przesmycki (Miriam)}

13 Maeterlinck apparaît dès le début du mouvement de la Jeune Pologne grâce à l'étude de Przesmycki intitulée «Maurycy Maeterlinck i jego stanowisko we współczesnej poezji belgijskiej "(Maurice Maeterlinck et son statut dans la poésie contemporaine belge) parue en premier lieu dans la revue cracovienne "ŚWIAT» (1891) ${ }^{17}$ et ensuite sous forme d'introduction au Wybór pism dramatycznych (Choix de textes dramatiques, 1894) ${ }^{18}$. Cet ouvrage contient des traductions de poèmes tirés des Serres Chaudes ainsi que les pièces L'Hôte inconnu, Les Aveugles, les Sept princesses, Pelléas et Mélisande, et également un extrait assez important de La Princesse Maleine. Przesmycki agrémenta son étude de ses propres études, il participa ainsi à la création d'une image de la littérature étrangère qui deviendra le point de départ de la conscience symboliste moderne. La publication des textes de l'auteur belge eut lieu, il est important de le souligner, la même année que celle de la traduction des Fleurs du Mal ainsi que de la première traduction de la poésie de Rimbaud dans la presse cracovienne. Par conséquent, dès le début de la Jeune Pologne, apparaissent deux sources étrangères, l'une belge, l'autre française, auxquelles la littérature symboliste polonaise alla puiser à sa manière ${ }^{19}$. En outre, «le début livresque " de Maeterlinck dans la Jeune Pologne coïncide avec la naissance de la nouvelle génération de poètes : Kasprowicz (Poezje [Poésies], 1889), Kazimierz Przerwa-Tetmajer (Seria I Poezji, [Série I de Poésie] 1891, Seria II Poezji [Série II de Poésie], 1894), Antoni Lang (Pogrzeb Shelleya [L'Enterrement de Shelley], 1890) et Andrzej Niemojewski (Poezje [Poésies], 1891).

Dans son étude, Przesmycki situe le renouveau littéraire de la culture belge dans les années 1880 . Il y parle de la naissance et de la première phase de développement de la Jeune Belgique, résume les principales discussions idéologiques et les principes esthétiques de l'époque, présente un panorama de la vie littéraire dans deux centres, Louvain et Bruxelles, et décrit également les nouveautés modernistes de la poésie symboliste d'Émile Verhaeren et de Georges Rodenbach. Le regard porté sur les faits occasionne un discours sur Maeterlinck qui, quel qu'en soit le thème principal, peut être également lu comme un guide historique fiable sur la première décennie du modernisme belge. Przesmycki créa en fait la première monographie étrangère sur la littérature belge moderne fin de siècle.

15 L'intention principale du critique était, bien entendu, d'offrir une vision détaillée de la création de jeunesse de Maeterlinck, depuis ses débuts poétiques (les Serres Chaudes, 1889) et les prémices de ses nouvelles (Onirologie, 1889), jusqu'après sa première pièce, Pelléas et Mélisande (1892). Le chercheur, qui se faisait également critique, s'attarda également sur les traductions de l'écrivain belge (il cite celle des Ornement des Noces Spirituelles, les écrits mystiques de Jan van Ruysbroeck) auxquelles il ajouta un avant-propos du traducteur, édité originellement séparément dans la revue parisienne « LA REVUE GÉNÉRALE » ${ }^{20}$ en 1889. Przesmycki ne se limita pas à la création fictionnelle et aux traductions popularisantes, il introduisit également dans son discours quelques extraits des commentaires 
philosophiques et littéraires que l'écrivain belge avait sporadiquement publié dans d'éminents magasines littéraires tels que « L'ART MODERNE » et « LA JEUNE BELGIQUE ». Grâce à cette solide préparation bibliographique, les lecteurs cracoviens et varsoviens (respectivement en 1891 et 1894) eurent la chance de faire connaissance avec les autocommentaires et les textes programmatiques de Maeterlinck qui avaient presque disparu de la conscience de la critique littéraire internationale, voilés et dominés qu'ils étaient par le succès des essais Le Trésor des Humbles (1896) et La Sagesse et La Destinée (1898). Cela vaut la peine d'être rappelé, étant donné que dans le domaine littéraire belge, et plus largement européen, la réédition de ces extraits d'articles ne parut qu'en 1985 et ce, grâce aux efforts de l'éditeur Stefan Gross ${ }^{21}$. En plus des textes publiés, des résumés et des citations des voix les plus importantes de la critique française (Octave Mirbeau) et belge (l'article de Verhaeren sur les Serres chaudes) ont été édités.

C'est justement la publication des premiers articles de Maeterlinck, de ces « inédits », qui est devenue, grâce à la critique polonaise, une source de reconstruction de la conception du monde et du programme littéraire adoptés par l'écrivain. Nous apprenons donc que Maeterlinck a créé une nouvelle conception de l'homme, basée sur le dualisme entre le matériel et le transcendantal. Il a cherché l'être originel dans la nature humaine, et le décrivit sous le vocable « d'archétype ». Cet archétype se manifeste avant tout dans la vie $\mathrm{du}$ subconscient. Les états frontaliers et irrationnels étaient, pour Maeterlinck, les plus importantes épiphanies de cette profondeur : les rêves, les fantasmes, l'instinct spirituel ( "la lumière intérieure »), la maladie et «le miracle de la mort», comme l'a qualifiée Maeterlinck dans La Confession du poète. Przesmycki décrit ces manifestations de la vie intérieure comme des «signes mystiques de la nature humaine »; il considérait comme leur contexte scientifique le plus proche le développement de la psychopathologie moderne, de la psychologie de l'inconscient, de la parapsychologie (dans ce dernier cas, il se revendiquait des conceptions de la psychologie transcendantale du chercheur allemand Carl du Prel), mais également le développement de la physique moderne qui avait découvert et tentait d'explorer la matière invisible et ses radiations. Tels la science moderne et le savoir qui s'ouvraient de plus en plus à une réalité profonde qu'une connaissance empirique ne pouvait appréhender, l'œuvre de Maurice Maeterlinck, per analogiam, s'ouvrit sur une sphère invisible dans la nature et l'intérieur de l'homme. Przesmycki conclut comme ceci : la réaction mystique dans la science devait porter les fruits du mysticisme littéraire de l'écrivain.

Après avoir tracé le contexte intellectuel de l'époque et caractérisé le tournant antipositiviste qui formèrent le terreau des idées de Maeterlinck, Pzesmycki introduit des questions fondamentales de nature esthétique. C'est avant tout le symbole qui a retenu son attention, le symbole compris dans un sens large, comme un phénomène ontologique, psychologique et littéraire. Lorsque Przesmycki écrit que « l'homme de Maeterlinck » est « l'être dont la partie sensitive est en quelque sorte le symbole concret d'une autre partie, infinie et transcendantale » et que sa nature est « un maillon dans la chaîne infinie des innombrables formes et apparences de l'être originel ${ }^{22}$, il a à l'esprit une compréhension anthropo-philosophique du symbole selon lequel la matérialité et la conscience de l'homme sont des signes monistes de l'éternité de l'être. Lorsqu'à un autre endroit, il confirme que le symbole est une idée traduite en un équivalent visuel émotionnel, il comprend clairement le symbole dans un sens psychologique, comme une image mentale de l'idée. Il ajoute que la pensée imagée dépasse la conscience rationnelle et caractérise avant tout l'imagination des artistes ${ }^{23}$. 

théâtrale de Maeterlinck. Le symbole poétique est défini ici comme une représentation plastique de la substance inconsciente par analogie au monde sensoriel. C'est une sensation de confusion et d'obscurité déguisée en « une forme sensorielle » ${ }^{24}$. Przesmycki met l'accent sur l'innovation du vers libre maeterlinckien qu'il considère comme un signe graphique de l'expression de la vérité intuitive. Il attache une grande importance à la musicalité du mot poétique qui, se référant à l'intonation naturelle, rompt avec la métrique de la tradition parnassienne. compréhension extensive et exclusive du symbole. Au sens large, le symbole est un drame en tant que texte littéraire, un être composé de mots. La mise en scène, concrétisation de la polysémie d'une œuvre littéraire, condamne le symbole à l'univocité. La tentative de se libérer de la pression de cette dialectique entre le texte-symbole et la représentation, comprise comme une réduction sémantique et picturale, a conduit le dramaturge belge à utiliser la technique symboliste. Ici, le symbole est

1) créateur d'ambiance du paysage romantique qui, dans les œuvres scéniques de Maeterlinck, possède un contenu fataliste,

2) une conception antipsychologique du héros (un fou ou un mannequin),

3) une fable intemporelle inspirée des contes et des légendes populaires qui rompt ostensiblement avec la mimésis du drame naturaliste. Przesmycki attire également l'attention sur la fonction symbolique de la langue dans La Princesse Maleine. Cette langue, subordonnée à l'expression des "états mystiques » de la conscience, se transforme en répétitions et pauses intentionnelles qui éloignent la couche dialogique du style de la communication quotidienne. Le critique conclut finalement en paraphrasant les mots célèbres de Rémy de Gourmont : « Le grand art, l'art essentiel, l'art immortel était, et est toujours, symbolique : il cache des éléments de l'infini derrière des analogies sensorielles, il dévoile des horizons immenses et extrasensoriels. $»^{25}$

Par son intérêt porté aux faits et par ses interprétations, l'étude de Przesmycki apporte des informations objectives sur le thème du mouvement symboliste dans la jeune poésie belge et précise les principales caractéristiques de la nouvelle éthique dans le domaine du théâtre à l'époque du «triomphe du naturalisme ». Selon Maria Podraza-Kwiatkowska, cette étude constitue l'une des théories et des pratiques d'écriture symboliste les plus importantes dans le premier stade de développement de la critique littéraire de la Jeune Pologne. Cependant, les chercheurs oublient souvent que le symbolisme n'est pas le seul et, loin s'en faut, l'ultime qualificatif esthétique aux yeux de Przesmycki, l'interprète des sources d'imagination de l'écrivain belge.

Le deuxième et ultime qualificatif est, d'après le critique, le terme " réalisme large ». Par « réalisme large », il entend la nouvelle direction prise par la création artistique dont la tâche est de montrer les dimensions non empiriques de la réalité, celles qui sont invisibles mais existent dans les faits. Il s'agit de la réalité qui, à l'intérieur de l'homme, s'étend au-delà des barrières de la logique et de la raison (surréalité / catégorie du surréel *) et qui, dans le monde objectif, équivaut aux phénomènes naturels inexplorables, traditionnellement reconnus comme surnaturels (catégorie du naturel inexploré*). D'après Przesmycki, "le mysticisme moderne " s'étend sur ces deux plans dans l'œuvre de Maeterlinck. Dans son esquisse du développement de la littérature européenne à l'époque du tournant moderniste, Przemycki considère ni plus ni moins que le naturalisme 
spirituel de Joris-Karl Huysmans constitue le contexte le plus proche pour un réalisme compris de la sorte.

Le progrès scientifique semble en effet confirmer la réalité des vérités cachées dans les anciens préjugés et les anciennes croyances, et quant au domaine traditionnel du surnaturel, il est remplacé par la recherche d'un " autre monde intérieur » qui se cache dans l'âme, dans l'hypostase de l'inconscient et du spirituel. Ce parallèle distinct entre le triomphe de l'empirisme dans les sciences positivistes et le triomphe de la fiction réaliste qui lui correspond dans la littérature moderne et surtout, comme le montre l'exemple de Huysmans, dans la prose narrative moderne, permet d'émettre l'hypothèse selon laquelle Przesmycki concrétisa, sur base de l'œuvre de Maeterlinck, la catégorie du fantastique moderniste caractéristique du "roman symboliste » moderne (de Jean Moréas), et ce en plus du symbolisme qu'il avait déjà concrétisé dans la poésie et le théâtre.

Il n'y a qu'un pas entre cette conception du « réalisme large » et celle, plus tardive, du "fantastique réel »*, inventé en 1967 par Franz Hellens. Par fantastique, il comprenait "l'explosion ou l'illumination du réel inconnu "*, "la levée du réel exaltant ${ }^{28}$.

Dans la prose de jeunesse de l'auteur belge, et plus précisément dans le récit Onirologie (1889), inspiré, comme l'ont plus tard remarqué les critiques, par la prose fantastique de Guy de Maupassant, Przesmycki trouve la confirmation de l'appartenance des premières œuvres de Maeterlinck au « réalisme large ». En conclusion, nous pouvons affirmer que le « réalisme large » de Przesmycki désigne en réalité le fantastique littéraire qui, selon le philosophe français Alain Chareyre-Méjan, correspond à la présentation littéraire du "comble du réel ${ }^{* 29}$. Celui-ci apparaît en même temps que prennent vie des phénomènes nouveaux, jusqu'alors inconnus, qui provoquent fascination et crainte.

Il s'avère donc que l'étude de Przesmycki se livre à un double style de lecture et d'interprétation. Elle constitue, sans aucun doute, une interprétation inestimable et unique du symbolisme dans la création de Maeterlinck ainsi qu'une présentation de la catégorie du fantastique littéraire (bien que cette idée n'apparaisse pas une seule fois dans le discours du critique) caractéristique de la prose narrative de la période symboliste.

La publication de Przesmycki provoqua, en Pologne, le premier débat animé au sujet de l'œuvre de l'écrivain belge et de la tâche qui attendait la littérature à la période de la « révolte moderniste». Pour la critique conservatrice, cette discussion sur la littérature polonaise ne touchait que superficiellement l'auteur des Serres chaudes et ses premières pièces de théâtres. C'était, en réalité, une occasion de nier les nouveaux courants littéraires venant de l'occident, tels que le décadentisme et le symbolisme ${ }^{30}$. La réaction négative à l'écriture de Maeterlinck émanait de ses adversaires principaux : Teodor JeskeChoiński et Józef Wessenhoff, appuyés par l'autorité scientifique de Max Nordau. Dans Dégénérescence (1892-1893), livre qui connut un certain succès, celui-ci, motivé par la réaction idéaliste des autres représentants de la culture européenne de la fin du XIXe, qualifia l'auteur belge d'écrivain psychopathe.

\section{La voix de Stanisław Przybyszewski}

Lorsque nous nous intéressons de plus près à l'époque de la Jeune Pologne, nous pouvons remarquer un enchaînement de faits relatifs à la réception de l'œuvre de Maeterlinck, et ce, à la fois dans le discours critique au sens large et dans l'événement théâtral. En 1899, 
eut lieu, au théâtre Municipal Juliusz Słowacki de Cracovie, la célèbre première d'Intérieur, dans une mise en scène de Tadeusz Pawlikowski ${ }^{31}$. La pièce fut précédée d'une lecture, faite par Przybyszewski, intitulée Mistyka a Maeterlinck (La mystique et Maeterlinck). La représentation de la pièce fut considérée par le critique comme le commencement du triomphe du théâtre de la Jeune Pologne. Ce triomphe coïncidait donc avec l'intronisation du théâtre symboliste maeterlinckien sur la scène polonaise.

La mise en scène d'Intérieur correspondait à la vision du nouveau théâtre de Maeterlinck.

Pawlikowski, le metteur en scène, insista sur le conventionalisme de la pièce, il l'exprima par une scénographie synthétique ainsi que par la réduction de la psychologie dans le jeu des acteurs. Comme le suggère le titre de la pièce, les acteurs se comportaient de manière anormale et hiératique, tels des marionnettes. Une attention particulière fut portée à la gestuelle des acteurs. Le titre polonais de la pièce Intérieur comportait cette indication: drame pantomime. La scène était conforme aux indications des didascalies, on y voyait une maison dans un jardin, éclairée de l'intérieur par une lumière vive. Les personnages du Vieillard et de l'Étranger se tenaient au premier plan, plongés dans un clair-obscur. Cette composition scénique soulignait la dialectique symboliste de la lumière et de l'obscurité, de la vie et de la mort. Cette dialectique est caractéristique d'Intérieur et, de façon générale, de toutes les pièces tragiques de Maeterlinck.

Ce souci de produire une atmosphère de fatalité se rencontrait également dans la disposition de la salle. Tous les spectateurs étaient assis au même niveau dans le parterre, face aux acteurs. Cet agencement avait pour but de supprimer la tridimensionnalité, et donc la réalité des personnages, et de créer une impression d'immobilité " picturale » des événements montrés. L'espace alloué aux spectateurs avait été réduit. Un rideau noir séparait, sur toute la hauteur, les premiers rangs du reste de la salle. Ce rideau servait à intensifier l'atmosphère funèbre. Grâce à ces opérations scéniques, la représentation cracovienne fut, comme l'affirma Stykowa, un spectacle inhabituel, une sorte de célébration scénique de la création du poète belge ${ }^{32}$. Voici quelques mots d'une critique qui reflète très bien l'aura de fête qui accompagna la première : «La représentation eut lieu sur invitation, celle-ci ne fut pas adressée à des hauts dignitaires mais aux représentants du monde artistique et littéraire ainsi qu'aux fervents défenseurs des nouveaux courants. Être reçu à ce mystère d'initiés était réellement un privilège. $»^{33}$

Ajoutons ici que la mise en scène mémorable d'Intérieur était liée au projet de créer, dans la capitale culturelle de Galicie, "une scène libre», une institution théâtrale indépendante vouée à la promotion de la création théâtrale avant-gardiste. Cette entreprise réalisée sur le sol polonais était inspirée, d'une part, par la tradition du théâtre naturaliste français et allemand (Théâtre-Libre Antoine, Freie Bühne d'Otto Brahm) et d'autre part, par les réalisations contemporaines du théâtre symboliste d'Aurélien LugnéPoe et de Paul Fort. La création d'un "théâtre libre » sur le sol polonais n'aboutit pas, mais l'événement qu'avait été la représentation de la pièce de Maeterlinck laissa une empreinte durable dans l'esprit des gens de théâtre et des créateurs du drame moderne dans la littérature polonaise. Le premier drame de Maeterlinck, auquel appartient Intérieur, fut en effet le modèle littéraire suivi par les principaux dramaturges de la Jeune Pologne dans les années 1890. Wyspiański satisfit son "maeterlinckisme» dans Warszawianka (La Varsovienne, 1898), Przybyszewski dans ses pièces Gość (L'invité, 1899) et Śnieg (La neige, 1901). Roman Taborski ${ }^{34}$ fit remarquer qu'un peu plus tôt cela avait été le cas de Lucjan Rydel dans Matka (La mère, 1893) et de Przerwa-Tetmajer dans Sfinks (Le sphinx, 1893), qui dévièrent sur le chemin du drame symboliste atmosphérique grâce aux 
traductions des drames de Maerterlinck publiées en Pologne dès 1891. Le drame maeterlinckien fut donc un stimulant important qui présida à la naissance du théâtre symboliste polonais et de sa première phase de développement.

31 La lecture que fit Przybyszewski fut imaginée comme une partie intégrante de la conception scénique. Elle se fit sous une lumière tamisée, la voix de l'écrivain, surgissant de l'obscurité, anticipait celles des personnages du Vieillard et de l'Étranger qui, dans la pièce, étaient eux-mêmes plongés dans l'obscurité de la nuit. Le discours de celui qui écrivit Requiem aeternam (La Messe des Morts, 1893) et fut plus tard rédacteur de la revue cracovienne "ŻYCIA », suggérait que ce "mot d'introduction" se dégageait de "l'intérieur » même de la philosophie et de la pensée esthétique de Maeterlinck. Il ne faisait que suggérer, car enfin la lecture de Przybyszewski avait bien peu de rapport avec la représentation qui suivit et avec la création de l'auteur belge en général. L'orateur commença par présenter les principales caractéristiques du symbolisme dans « la poésie dramatique». Il souligna que le théâtre moderne s'efforçait de dépeindre les états psychiques de l'homme et, de ce fait, s'éloignait de l'imitation des événements de la vie. L'action extérieure, perçue comme insignifiante, se dérobe devant la présentation des états d'âme et des pressentiments vagues. L'imitation des " états d'âme » constitue donc le fondement philosophique de ce "nouveau théâtre", "car elle conduit à la connaissance de la vie $\aleph^{35}$. Après une présentation sommaire du concept de théâtre symbolique, Przybyszewski en vint au développement de ses conceptions de l'âme, sujet qui le fascinait depuis longtemps. En s'appuyant sur les sciences occultes et la parapsychologie, il remarqua que l'âme humaine se caractérisait par sa nature indépendante et qu'elle préexistait à la naissance de l'individu. Il est possible de découvrir cette préexistence à condition que l'individu, sur le chemin de la connaissance extra-sensorielle, trouve en lui l'horizon caché de l'éternité. Cette éternité n'a cependant pas grand chose en commun avec la religion transcendantale de Maeterlinck, qui, dans Le Trésor des Humbles, décrit l'âme comme une part de divinité reposant à l'intérieur de l'homme. Selon Przybyszewski, et en cela il s'éloignait du spiritualisme chrétien de Maeterlinck, l'âme est restée esclave des lois de la nature. Les instincts biologiques en sont la trame profonde, ils paralysent les droits de la raison et privent l'être humain de libre volonté. Pour Maeterlinck, l'âme est un élément surnaturel; pour Przybyszewski, l'âme est par essence une natura naturans intériorisée. Przybyszewski trouva dans les pièces de Maeterlinck, en forçant, et à tort, la confirmation de sa conception matérialiste de la fonction de la nature. Il affirmait en effet que la nature avait une influence directe sur les actions des héros. Il confondait cependant le déterminisme naturaliste et la fonction atmosphérique et symboliste du paysage qui, dans les œuvres de l'écrivain belge, accompagne les états d'âme des héros et devient une visualisation de leurs craintes, de leurs espoirs ou annonce des événements motivés par des forces métaphysiques (et les lois de la nature qui n'ont rien de commun avec elles). La mystique et Maeterlinck,lu en 1899, apparut donc comme une présentation «épidermique » de l'écriture et de la vision du monde proposée par l'auteur d'Intérieur. En réalité, c'était déjà le noyau de son propre programme esthétique que Przybyszewski devait présenter, sous une forme élargie, dans son étude intitulée $O$ dramacie $i$ scenie (Sur le théâtre et la scène, 1905). Il convient toutefois de noter que le manque d'objectivité donne un certain attrait à l'interprétation qui s'y dévoile. La réception objective se transforme ici en une interprétation qui, comme le voulait Jauss, est continuellement marquée par la partialité. Peut-être a-t-elle été lue comme une inversion spirituelle spécifique de l'auteur du Trésor des Humbles. 

dans la littérature polonaise fut la plus haute. Le futur proche le prouvera. Elle fut à la fois un point d'épanouissement et de déclin. Les décennies suivantes devaient apporter les premiers signes de la réévaluation de son œuvre. l'esthétique symboliste fut doublement attaquée. Les deux fois, l'initiateur de cette attaque fut Brzozowski. En 1903, dans la recension de la représentation varsovienne de Monna Vanna, il critiqua le fond philosophique et la passivité de l'esthétique maeterlinckienne. Il lui opposait un art basé sur l'affirmation de la force de la vie et de l'engagement dans les problèmes sociaux ${ }^{38}$. Le rejet de Maeterlinck était, dans un certain sens, une introduction à la querelle qui opposa Brzozowski à Przesmycki, alors rédacteur de « CHIMERA » et qui devint, un an plus tard, la célèbre campagne journalistique menée contre les idéaux de l'art lancé par "l'AlmANACH» de Miriam. Une introduction seulement, car le profil esthétique de "CHIMERA », tout comme les idées du rédacteur sur l'art, furent, comme en témoignent l'analyse précédente, un développement conséquent du premier contact de Miriam avec l'œuvre symboliste de l'écrivain belge.

L'étude de Brzozowski fut dictée également par d'autres circonstances. En 1903, les éditions du «PRZEGLĄD TYGODNIOWY», la revue la plus lue parmi les périodiques polonais $\mathrm{du}$ tronçon russe, commencèrent à sortir sur le marché du livre la première série des Écrits de Maurice Maeterlinck.CEuvresdramatiques et scientifico-éthiques. Rien qu'au cours de l'année 1903, quatre tomes furent publiés. Respectivement : t. 1 : Monna Vanna, t. 2 : La Vie des abeilles, t. 3: Le Temple enseveli, t. 4: La Sagesse et la destinée. Tous ces livres «scientifico-éthiques» ont été traduits par Róża Centnerszwerowa. Il est important 
d'ajouter ici que Le Trésor des Humbles est sorti en version polonaise au même moment, tout comme ce fut le cas pour un choix d'extraits du premier livre d'essais de Maeterlinck intitulé 0 Kobiecie. Dobroć niewidzialna. Tragizm codzienny (À propos de la femme. La bonté invisible. La tragédie quotidienne). En douze mois à peine fut ainsi publiée une imposante collection. 1903 peut donc être appelée l'année du triomphe de la lecture de Maeterlinck, triomphe auquel Przesmycki et Przybyszewski avaient préalablement préparé le terrain. Mais le succès aux yeux d'un public de masse ne va pas pour autant de pair avec la reconnaissance de la critique. Et critique, Brzozowski l'était, lui qui avait développé de nouveaux horizons pour la littérature de la Jeune Pologne. Si l'on s'en réfère aux fait, il est évident que la question ne touchait pas les destinées scéniques d'une pièce au sujet d'une princesse italienne, mais bien la résistance à la vague de popularité monstre du Maeterlinck-philosophe qui commençait justement, de par la presse et les pièces de théâtre, à être connu du lecteur intellectuel en Pologne. Brzozowski savait que derrière la richesse de la pensée de Maeterlinck, il n'y avait pas seulement le «PRZEGLĄDY TYGODNIOWY » ou une réponse à des goûts nationaux. Déjà à partir de la moitié des années 1890, le Maeterlinck-essayiste avait captivé un large public en France (en 1901, à Paris, il y eut vingt-et-une rééditions du Trésor des Humbles) ainsi qu'en Allemagne (l'édition intégrale du Trésor des Humbles y était déjà publiée en 1898) mais également en Autriche où de larges extraits de ses essais avaient été largement diffusés dans les périodiques modernistes. L'attaque menée contre Maeterlinck devait donc servir, toutes proportions gardées*, à réduire l'attraction exercée par l'évangile mystique de l'auteur belge. Cet évangile enseignait, comme le voulait son adversaire, la sensiblerie, une vision du monde efféminée, la résignation stoïque et la formation du cervelet «des tourterelles modernisées $»^{39}$.

En résumé, nous pouvons affirmer que cette campagne anti-maeterlinckienne servit deux buts : premièrement, surmonter la philosophie et l'esthétique symboliste qui avait fleuri sur le tronc de la décadence (la critique sévère de « GıOS » prit position contre la réalité littéraire). Deuxièmement, dépasser le programme de vie proposé par Maeterlinck dans ses livres-essais (Brzozowski s'est battu pour un idéal de "vie », considéré par Nietzsche comme un acte de courage et une auto-réaction ferme).

Brzozowski attaqua le «manque de sincérité » de Maeterlinck. Celui-ci devait consister en la popularisation d'idées et de certitudes en lesquelles l'auteur lui-même ne croyait pas. Aux yeux de Brzozowski, Maeterlinck n'était pas vraiment un mystique, il était simplement un positiviste "encapuchonné " qui, grâce à sa rhétorique mystique, s'efforçait de donner l'impression du mystère immanent de la vie humaine. Maeterlinck fut accusé d'inconséquence : l'écrivain belge alla jusqu'à la frontière de la connaissance mais laissa le lecteur au bord du mystère, il s'est ensuite résigné à explorer des domaines inconnus, se contentant d'évoquant les auras du mystère avec sa propre phraséologie. Son œuvre apparaît comme une mystification du vrai mysticisme esthétique, une mystification qui pèche par verbalisme et manque d'authenticité. Brzozowski a peut-être raison lorsqu'il fait remarquer que Maeterlinck, malgré qu'il ait écrit à plusieurs reprises sur la vie de l'esprit, n'opère pas par la langue de l'âme, il parle toujours d'un point de vue d'intellectuel modeste.

D'autre part, Brzozowski n'aurait pas pu reprocher à l'œuvre de Maeterlinck ce qui ne s'y trouve pas, car cela a été consciemment rendu impossible par l'auteur d'Intérieur. «W duszE, jak w tę oto izbణ̨, zajrzeć nie można... » - c'est la phrase du Vieillard dans l'Intruse et le credo épistémologique de l'auteur. 
40 La deuxième raison de cette attaque était son "byzantinisme ", à savoir l'esthétique exclusive et la métaphysique qui ne prennent soi-disant pas en compte la problématique sociale. Pour Brzozowski, élevé au moralisme du premier Ibsen, au courage démasquant de Nietzsche, à la psychologie de Dostoevskij et de Przybyszewski, le premier «maitre » de sa jeunesse intellectuelle, l'atmosphèreet le programme apsychologique de Maeterlinck, tout comme l'axiologie du silence si caractéristique de l'écrivain, devaient apparaitre comme des signes vides. Brzozowski a opposé à Maeterlinck un art qui devait être « un redresseur de la vie sociale » et une source d'élévation spirituelle commune. «Byzantinistes! Une nouvelle forme d'art empreigne votre travail, mais vous ne pouvez pas la posséder. Une nouvelle époque s'ouvre dans l'art, celle de l'approfondissement, une source de forces pour toutes les âmes, une époque nouvelle, ferme, forte, sévère qui assure toutes les valeurs à sa manière. N'entendez-vous pas déjà les coups de marteau ?» ${ }^{41}$ Ajoutons par ailleurs que Brzozowski avait tort d'accuser Maeterlinck d'un manque d'intérêt pour les problèmes collectifs. Il n'a pas remarqué que dans ses essais, Maeterlinck n'était pas seulement " une femme incomprise ». Ce domaine de son écriture, à la différence de sa poésie et de son théâtre, est né, comme l'a prouvé Paul $\mathrm{Aron}^{42}$, du courant de «l'art social », lequel existait sur le sol belge à la même époque, et, au sein même de l'œuvre de Maeterlinck, elle évolua avec la tendance esthétique de «l'art pour l'art ».

41 La campagne menée contre Maeterlinck n'eut pas d'écho, contrairement à celle qui suivit tout de suite après. L'édition des œuvres de l'écrivain belge fut interrompue, non pas à cause de la polémique provoquée par le critique en chef de la rédaction de «GŁOS » mais en raison du manque de moyens financiers. Les années suivantes montreront que les priorités esthétiques et les points de vue de la Jeune Pologne avaient évolué. Maeterlinck fut déplacé hors du centre des débats littéraires et il bascula dans une phase close de l'histoire moderne de la littérature universelle. La révolution de 1905-1907 laissa les écrivains face à de nouvelles tâches et entraîna un retour du paradigme romantique de la création littéraire. De nouveaux noms apparurent: Bolesław Leśmian, Tadeusz Miciński, Wacław Berent. Bien qu'ils commencèrent sous l'égide du symbolisme, le premier continua de puiser dans la poésie russe et les deux autres évoluèrent vers l'expressionnisme. Pour les derniers arrivants de la Jeune Pologne, Zofia Nałkowska, Juliusz Kaden-Bandrowski, Stanisław Ignacy Witkiewicz (Witkacy) et Roman Jaworski, Maeterlinck faisait déjà partie du passé.

C'est dommage tant il est vrai que l'œuvre de Maeterlinck n'est pas monolithique, qu'elle connut un développement que ni Brzozowski ni ses adversaires de moindre envergure n'avaient remarqué. Depuis La Sagesse et la Destinée (1898), les textes populaires de l'écrivain belge évoluaient vers l'affirmation de la vie quotidienne, de ses charmes prosaïques et de ses moments de souffrance inévitables. Jadis fataliste, observant autour de l'effroi causé par le destin cruel qui atteint l'homme perplexe face à la mort ou l'amour malheureux et tragique, il commença, progressivement, et sous l'influence des écrits de Marc Aurèle, à accepter la nature humaine. Il se mit à clamer l'héroïsme de l'individu, l'idéal de « la vie-pour-soi » et le perfectionnement moral. Si les essais de l'auteur belge avaient éveillé un grand intérêt chez les écrivains et la critique professionnelle dans les deux premières décennies du $\mathrm{XX}^{\mathrm{e}}$ siècle en Pologne, Maeterlinck aurait eu la chance de devenir l'un des pères spirituels de la littérature du "courant prosaïque ", rejoignant ainsi Leopold Staff (à partir de Gałęzi kwitnĄcej [les branches florissantes], 1908) et la poésie d'Aleksander SzczĘsny. Il est intéressant de se demander à quoi aurait ressemblé la 
combinaison de KsiĘgi ubogich (Les livres des pauvres, 1911) de Kasprowicz et de l'apologie de la sagesse des gens simples que proposait LeTrésor des Humbles de Maeterlinck. Le futur Prix Nobel créa dans ses articles philosophiques une variante de la conscience populaire mystique que l'on pourrait qualifier, toutes proportions gardées, de franciscaine. Sous de nombreux aspects, elle répond à la une revalorisation poétique de la simplicité qui apparaissait alors dans la littérature polonaise. Lorsqu'en 1909 sortit la traduction de Maeterlinck. Étude critique ${ }^{43}$, le livre du critique allemand Monty Jacobs consacré aux principales lignes d'interprétation de cette évolution intérieure, le "nouveau " Maeterlinck n'éveillait déjà plus d'émotion parmi les coryphées de la littérature polonaise. Une autre raison de cet «effacement » de la pensée de Maeterlinck dans le principal courant de la littérature polonaise pourrait aussi être s'expliquer par la réception de l'œuvre du philosophe Henri Bergson qui se fit connaître dans la première décennie du XXe siècle. Elle remplaça par son autorité académique l'intuitionnisme naïf de l'auteur de La Sagesse et la Destinée.

Ainsi, la deuxième décennie de la Jeune Pologne ne profita pas des possibilités que laissait malgré tout l'œuvre de l'écrivain belge, l'image qui resta de lui était figée dans un schéma atmosphérique. Dans la littérature occidentale, Maeterlinck s'est vu octroyé la place honorable d'important écrivain-penseur qui, comme le souhaitait au début du XXe siècle Monty Jacobs ${ }^{44}$, combina des valeurs éloignées : l'élitisme et le révisionnisme de Nietzsche avec le christianisme de Tolstoj. En raison d'une évolution littéraire différente et d'une histoire turbulente, la Jeune Pologne ne parvint pas à combiner de tels éléments.

\section{NOTES}

1. Żurowski M., "Cieplarnie" Maurycego Maeterlincka czytane na przełomie stuleci (Les Serres chaudes de Maurice Maeterlinck lues au tournant du siècle), in : Symbolizm francuski i Młoda Polska. Studia $i$ materiały (Le symbolisme français et la Jeune Pologne. Études et matériaux) (Sous la direction de Chudak H.), Uniwersytet Warszawski. Instytut Romanistyki, Varsovie, 1994, pp. 65-97.

2. Stykowa M. B., Teatralna recepcja Maeterlincka w okresie Młodej Polski (La réception théâtrale de Maeterlinck dans la période de la Jeune Pologne), Zakład Narodowy im. Ossolińskich, Wrocław, 1980 ; « Maeterlinck w Młodej Polsce (mit dramaturga)» (Maeterlinck dans la Jeune Pologne [le mythe du dramaturge]), in : Wśród mitów teatralnych Młodej Polski (Parmi les mythes théâtraux de la Jeune Pologne) (Sous la direction de Sławińska I. et Stykowa M. B.), Wydawnictwo Literackie, Cracovie, 1983, $468 \mathrm{p}$.

3. Sławińska I., Młodopolska batalia o teatr (Le conflit à propos du théâtre dans la Jeune Pologne), introduction à : Sławińska I. \& Kruk S., Myśl teatralna Młodej Polski. Antologia (La pensée théâtrale de la Jeune Pologne. Anthologie), Wydawnictwa Artystyczne i Filmowe, Varsovie, 1966, 475 p.; Tragedia $w$ epoce Młodej Polski. $Z$ zagadnień struktury dramatu (La tragédie à l'époque de la Jeune Pologne. Études de la structure du drame), Towarzystwo Naukowe w Toruniu. Prace Wydziału Filologiczno-Filozoficznego, t. 1, z. 2, Toruń, 1947, pp. 147-161.

4. Słomkowska A., "Le paysage dans le théâtre de Maeterlinck ", in : "ANNALES DE LA FONDATION MAURICE MAETERLINCK », t. IX, Gand, 1963, pp. 18-54. 
5. Szturc Wł., Maurice Maeterlinck ou la dialectique entre l'extérieur et l'intérieur de l'homme, Frans Roggenstichting, Gand, 1994, 16 p.

6. Słomkowska A., Art. cit.; Jakóbczyk S., «Structures picturales dans les drames de Maurice Maeterlinck », in : «ANNALES DE LA FONDATION MAURICE MAETERLINCK », t. XVII, Gand, 1971, pp. 33-67 ; Stykowa M. B., « La Première polonaise de "Pelléas et Mélisande”: Cracovie, 1906 », in : « ANNALES DE LA FONDATION MAURICE MAETERLINCK", t. XXV, 1979, pp.47-63 (supplément paru dans La Bibliographie des traductions polonaises des drames de Maeterlinck); Szturc Wł., "Maurice Maeterlinck et la Jeune Pologne (1894-1914) », in : « ANNALES DE LA FONDATION MAURICE MAETERLINCK ", t. XXX, 1997, pp. 83-95 [texte fourni avec la liste Les pièces de Maeterlinck en Pologne (1894-1918). Représentations choisies].

7. Les termes marqués d'un astérisque sont en français dans le texte (NdT).

8. Son premier recueil d'essais, Le Trésor des Humbles (Mercure de France, Paris, 1896) a été publié en 1898 en Allemagne (Leipzig) sous le titre Der Schatz der Armen, dans une traduction de Friedrich von Oppeln-Bronikowski. Ses illustrations et sa conception typographique, dues à Melchior Lechter, sont de style néo-germanique. Cet ouvrage donna naissance au style bibliophilique appelé « Old Morris », lié à la tradition anglaise du «beau livre ». Voir : Wais K., Maurice Maeterlinck, initiateur de Poètes allemands (traduit de l'allemand par Rita Gailliard), in : « SYNTHÈSES. REVUE INTERNATIONALE ", nº 195, 1962, p. 145.

9. Voir : «ANNALES DE LA FONDATION MAURICE MAETERLINCK », Gand, 1999, t. XXXI : Maeterlinck et les arts plastiques (Draguet M., « Du silence en peinture. Khnopff et le théâtre de l'image », pp. 57-68 ; Cantoni D., «La Picturalité du premier théâtre de Maeterlinck : Jessie King, les Nabis et quelques autres », pp.121-156; Pedretti-Mahler M.-A, «Au commencement était la glaise... L'Art de Georges Minne dans la démarche poétique de Maurice Maeterlinck de 1886 à 1889 ", pp. 179-247 ; Le tome se termine par une liste des «Illustrateurs des œuvres de Maurice Maeterlinck », pp. 248-260).

10. Stykowa M. B., Teatralna recepcja Maeterlincka..., Op. cit., pp. 127-131.

11. Juszczak W., Teksty o malarzach. Antologia polskiej krytyki artystycznej, 1890-1918 (Textes sur les peintres. Anthologie de la critique artistique polonaise, 1890-1918), Zakład Narodowy im. Ossolińskich, Wrocław, 1976, 478 p.

12. Les Peintres de l'âme. Le Symbolisme idéaliste en France, catalogue d'exposition « Les Peintres de l'âme ", Musée d'Ixelles, Bruxelles, 15 octobre - 31 décembre 1999 (Sous la rédaction de JumeauLafond J.-D., Gand / Anvers, 1999, pp. 10-25.

13. Grzegorz-Paweł BĄbiak a récemment écrit sur le salon plastique « CHIMERA » dans une vaste monographie consacrée aux textes de Przesmycki intitulée Metropolia i zaścianek. $W$ krĘgu „Chimery” Zenona Przesmyckiego (La métropole et le hameau. Autour de «CHIMERA » de Zenon Przesmycki), Nakładem Wydziału Polonistyki Uniwersytetu Warszawskiego, Varsovie, 2002, pp. 31-35 \& 52-55.

14. Bibliografia zawartości „Życia” warszawskiego i krakowskiego, „Strumienia” oraz "Chimery” (Bibliographie du contenu de „Życia ” à Varsovie et à Cracovie, de „Strumienie” et de „Chimera ") (Sous la direction de BĄbiak G.-P., Zakład Graficzny Uniwersytetu Warszawskiego, Varsovie, 2000, 175 p. S'y trouve la liste des illustrations dans chaque périodique.

15. Przybyszewski St., Exegi monumentum, [introduction à:] Wyspiański St., Dzieła malarskie (๕uvres picturales), texte écrit par Żuk-Skarszewski T., nakładem Instytutu Wydawniczego "Bibljoteka Polska", Bydgoszcz, 1925, 471 p.

16. Jauss H.R., Estetyka recepcji $i$ komunikacja literacka (Esthétique de la réception et communication littéraire) (traduit de l'allemand par Barbara Przybyłowska), in:Antologia zagranicznej komparatystyki literackiej (Anthologie de littérature comparative étrangère) (Sous la direction de Janaszek-Ivanićkovej H., Instytut Kultury, Varsovie, 1997, pp.164-175 (Première publication). Jauss H. R., «Esthétique de la Réception et Communication Littéraire", in: 
Proceedings of the IXth Congress of the International Comparative Literature Association, Literary Communication and Reception (Sous la direction de Konstantinović Z. et Neumann M.), Verlag des Instituts für Sprachwissenschft der Universität Insbruck, Insbruck, 1980, t. II, pp. 15-25.

17. Przesmycki Z., «Maurycy Maeterlinck. Stanowisko jego w literaturze belgijskiej», in : «Świat », 1891, n 3, pp. 58-62; n 4, pp. 90-92; n 5, pp. 112-117; n 6, pp. 134-138; $n^{\circ} 7$, pp. $162-164 ; n^{\circ} 8$, pp. $194-196 ; n^{\circ} 9$, pp. 212-213; n 10, pp. 237-241; $n^{\circ} 11$, pp. 261-263; $n^{\circ} 12$, pp. 288-289; n 14 , pp. $339-340 ; n^{\circ} 18$, pp. 432-435; n² 1 , pp. 506-509; $n^{\circ} 22$, pp. ${ }^{\circ} 537-538 ; n^{\circ} 23$, pp. 556-560; n²4, pp.585-592. Information tirée de PrzesmyckiZ. (Miriam), Wybór pism krytycznych (Choix de textes critiques), (Textes établis par KorzeniewskaE.), t. I, Biblioteka Studiów Literackich, Cracovie, 1967, p. 362.

18. Maeterlinck M., Wybór pism dramatycznych. Gość nieproszony. Ślepcy. Siedm królewien. Pelleas $i$ Melisanda (Choix de textes dramatiques. L'Hôte inconnu. Les Aveugles. Les Septes princesses. Pelléas et Mélisande.), traduit et introduit par Przesmycki Z. (Miriam), Éd. S. Lewental, Varsovie, 1894, pp. V-CXXII. J'utilise ici la réédition faite à Wrocław par les éditions WacBagiński et Fils (sous la direction de Kolbuszewski J., 1994.) Dans le livre, le titre de l'étude a été modifié suite à un élargissement significatif du contenu d'origine de « Maurycy Maeterlinck. Stanowisko jego w literaturze belgijskiej i powszechnej » (Maurice Maeterlinck. Son statut dans la littérature belge et universelle).

19. Il ne faut pas oublier que ce sont les travaux critiques antérieurs qui ont préparé le terrain à Maeterlinck et à la poésie de Baudelaire. Przesmycki a écrit une étude en plusieurs parties sur le développement de la poésie française du XIX ${ }^{\mathrm{e}}$ siècle : Profile poetów francuskich (Profils de poètes français), in: «ŻYCIE», Varsovie, 1888. Le lecteur polonais pouvait s'informer sur l'art de Baudelaire grâce, entre autres, aux développements analytiques de Waleria MarrenéMorzkowska et du critique littéraire Antoni Lange.

20. L'Ornement des Noces Spirituelles, de Ruysbroeck l'Admirable, traduit du flamand et accompagné d'une introduction de Maurice Maeterlinck, Paul Lacomblez, Bruxelles, 1891, $297 \mathrm{p}$.

21. Maeterlinck M., Introduction à une psychologie des songes. (1886-1896)", Textes réunis et commentés par Gross St., Labor, Bruxelles, 1989.

22. Przesmycki Z., «Maurycy Maeterlinck. Stanowisko jego w literaturze belgijskiej i powszechnej ", in : Maeterlinck M., Wybór dramatów (Choix de pièces), traduit et introduit par Przesmycki Z., Wydawnictwo S. Lewentala, Varsovie, 1894, p. LXXIII.

23. Ibid., pp. XLII-XLIII.

24. Ibid., p. XLIII.

25. Ibid., p. LXV.

26. Przesmycki Z., «Maurycy Maeterlinck. Stanowisko jego w literaturze belgijskiej i powszechnej », Art. cit., p. LXXIX.

27. Ibid., p. XCIII.

28. Hellens F., Le Fantastique réel, Sodi, Bruxelles/Amiens, 1967, pp. 13, 16.

29. Chareyre-Méjan A., Le Réel et le fantastique, L'Harmattan, Paris, 1999, p. 124.

30. Parmi les personnes célèbres qui étaient contre, il convient de citer, d'après Maria PodrazaKwiatkowska: Józef Weyssenhoff, Nowy fenomen literacki (Maurycy Maeterlinck i dekadentyzm symboliczny) (Nouveau phénomène littéraire [Maurice Maeterlinck et le décadentisme symboliste]), in : «BIBLIOTEKA WARSZAWSKA », Varsovie, 1891, ainsi que Jeskie-Choiński T., Rozkład w życiu i literaturze. Studium (Décomposition dans la vie et la littérature. Étude), Wydano nakładem drukarni « Wieku », Varsovie, 1895. Voir Programy i dyskusje literackie okresu Młodej Polski. Antologia (Programme et débats littéraires à l'époque de la Jeune Pologne) (rédigé et introduit par PodrazaKwiatkowska M.), Ossolineum, Wrocław, 2000, chapitre intitulé «Wokół twórczości Maeterlincka » (Autour de l'œuvre de Maeterlinck), pp. 460-482.

31. Stykowa M. B., " Nowatorskie inscenizacje "WnĘtrza" " (Mise en scènes novatrices d'Intérieur ), in : Teatralna recepcja Maeterlincka..., Op. cit., pp. 40-66. 
32. Ibid., p. 45.

33. Ibid., p. 44.

34. Kulczycka-Saloni J. et alii, Literatura polska. Młoda Polska (La littérature polonaise. La Jeune Pologne), PWN, Varsovie, 1991, p. 185.

35. Stykowa M. B., « Nowatorskie inscenizacje "WnĘtrza" ", Art. cit., p. 57.

36. Les informations sont données par Palleske S. O., Maurice Maeterlinck en Allemagne, Société d'Édition « Les Belles Lettres », Paris, 1938, p. 24.

37. Gorceix P., "Maeterlinck et Hermann Bahr», in: Maurice Maeterlinck. Le Symbolisme de la différence, Paris, 2000, p. 215.

38. Brzozowski St., Maurycy Maeterlinck ( $Z$ powodu wystawienia "Monna Vana» w Warszawie) (Maurice Maeterlinck. En raison de la mise en scène de "Monna Vana » à Varsovie), in : «GŁOS ", 1903 : Programy $i$ dyskusje literackie okresu Młodej Polski. Antologia (Programmes et discussions littéraires de l'époque de la Jeune Pologne. Anthologie), Op. cit., pp. 472-482.

39. Ibid., p. 476.

40. Brzozowski St., « Maurycy Maeterlinck (z powodu wystawienia „Monny Vanny” w Warszawie) „(Maurice Maeterlinck [en raison de la représentation de „Monna Vanna » à Varsovie]), in : Programy i dyskusje literackie okresu Młodej Polski, Op. cit., pp. 475-476.

41. Ibid., p. 482.

42. Aron P., Les Écrivains belges et le socialisme (1880-1913). L'expérience de l'art social: d'Edmond Picard à Émile Verhaeren, Éditions Labor, Archives du Futur, Bruxelles, 1985, p. 131.

43. Monty-Jacobs, «Maeterlinck. Studium krytyczne »(Maeterlinck. Étude critique), traduit de l'allemand par Maria Kreczowska, Biblioteka Dzieł Wyborowych, Drukarnia Granowskiego i Sikorskiego, Varsovie, 1909. Édition allemande : Maeterlinck. Eine kritische Studie zur Einführung in seine Werke, Eugen Diederichs, Leipzig, 1901.

44. Ibid., p. 92.

\section{INDEX}

Index géographique : Pologne

Index chronologique : entre-deux-guerres, fin de siècle, XIXe siècle, XXe siècle

Mots-clés : littérature belge, littérature polonaise

\section{AUTEURS}

\section{DARIUSZ DZIURZYŃSKI}

Professeur à l'Université de Varsovie 